\title{
Uncoupling neuronal death and dysfunction in Drosophila models of neurodegenerative disease
}

\author{
Amit K. Chouhan ${ }^{1 \dagger}$, Caiwei Guo ${ }^{2 \dagger}$, Yi-Chen Hsieh ${ }^{3 \dagger}$, Hui Ye ${ }^{3 \dagger}$, Mumine Senturk ${ }^{4 \dagger}$, Zhongyuan Zuo ${ }^{3}$, Yarong $\mathrm{Li}^{1}$, \\ Shreyasi Chatterjee, Juan Botas ${ }^{3}$, George R. Jackson ${ }^{1,5,6}$, Hugo J. Bellen ${ }^{2,3,4,7,8}$ and Joshua M. Shulman ${ }^{1,2,3,4,8^{*}}$
}

\begin{abstract}
Common neurodegenerative proteinopathies, such as Alzheimer's disease (AD) and Parkinson's disease (PD), are characterized by the misfolding and aggregation of toxic protein species, including the amyloid beta (Aß) peptide, microtubule-associated protein Tau (Tau), and alpha-synuclein (aSyn) protein. These factors also show toxicity in Drosophila; however, potential limitations of prior studies include poor discrimination between effects on the adult versus developing nervous system and neuronal versus glial cell types. In addition, variable expression paradigms and outcomes hinder systematic comparison of toxicity profiles. Using standardized conditions and medium-throughput assays, we express human Tau, Aß or aSyn selectively in neurons of the adult Drosophila retina and monitor age-dependent changes in both structure and function, based on tissue histology and recordings of the electroretinogram (ERG), respectively. We find that each protein causes a unique profile of neurodegenerative pathology, demonstrating distinct and separable impacts on neuronal death and dysfunction. Strikingly, expression of Tau leads to progressive loss of ERG responses whereas retinal architecture and neuronal numbers are largely preserved. By contrast, A $B$ induces modest, age-dependent neuronal loss without degrading the retinal ERG. aSyn expression, using a codon-optimized transgene, is characterized by marked retinal vacuolar change, progressive photoreceptor cell death, and delayed-onset but modest ERG changes. Lastly, to address potential mechanisms, we perform transmission electron microscopy (TEM) to reveal potential degenerative changes at the ultrastructural level. Surprisingly, Tau and aSyn each cause prominent but distinct synaptotoxic profiles, including disorganization or enlargement of photoreceptor terminals, respectively. Our findings highlight variable and dynamic properties of neurodegeneration triggered by these disease-relevant proteins in vivo, and suggest that Drosophila may be useful for revealing determinants of neuronal dysfunction that precede cell loss, including synaptic changes, in the adult nervous system.
\end{abstract}

Keywords: Alzheimer disease, Parkinson disease, MAPT, Tau, Amyloid-beta peptide, SNCA, alpha-synuclein, Neurodegeneration, Neurophysiology, Synapses, Animal model, Drosophila

\section{Introduction}

Alzheimer's disease (AD) [46] and Parkinson's disease (PD) [32] are common and incurable neurodegenerative disorders responsible for substantial cognitive and/or motor disability in the aging population. Both disorders are characterized at autopsy by widespread neuronal loss within the central

\footnotetext{
* Correspondence: Joshua.Shulman@bcm.edu

${ }^{\dagger}$ Equal contributors

'Department of Neurology, Baylor College of Medicine, Houston, TX 77030, USA

${ }^{2}$ Department of Neuroscience, Baylor College of Medicine, Houston, TX 77030, USA

Full list of author information is available at the end of the article
}

nervous system in association with aggregated and misfolded toxic protein species. AD is neuropathologically defined by the presence of amyloid plaques and neurofibrillary tangles, predominantly consisting of extracellular $A ß$ peptide deposits and intracellular filamentous aggregates of the microtubule associated protein Tau (MAPT/Tau), respectively. Similarly, intracellular inclusions of alpha-synuclein protein (SNCA/ $\alpha \mathrm{Syn}$ ) comprise the Lewy bodies that pathologically define PD. Importantly, since rare mutations in the amyloid precursor protein, MAPT, and SNCA genes all cause Mendelian, young-onset forms of dementia and/or 
parkinsonism, the misfolded and aggregated proteins that form pathological inclusions in $\mathrm{AD}$ and $\mathrm{PD}$ are causatively linked to disease pathogenesis. Further support comes from numerous transgenic animal models $[16,23]$ in which expression of Aß, Tau, or $\alpha$ Syn have been demonstrated to recapitulate neurotoxicity and often other clinicopathologic features relevant to human disease.

$\mathrm{AD}$ and $\mathrm{PD}$ are also similarly characterized by a prolonged, insidious onset in which absent or subtle symptoms precede more severe cognitive and motor disability and clinical recognition. While part of the age-dependent progression certainly relates to the cumulative toll of neuronal loss and failure of compensatory mechanisms, it is increasingly apparent that a period of cellular dysfunction precedes death [27]. For example, fibrillar $A ß$ pathology has been associated with altered measures of brain metabolism $[3,31]$ and connectivity [55] and these changes might potentially be explained in part by synaptic loss and dysfunction seen in experimental models. Similarly, clinicopathologic studies of PD suggest dopaminergic terminal dysfunction precedes nigral cell death [29], potentially contributing to early clinical manifestations of disease. Indeed, a current priority is to develop strategies to identify affected individuals earlier allowing potential therapeutic intervention before irreversible cell death [54].

Robust experimental models of neuronal dysfunction in $\mathrm{AD}$ and $\mathrm{PD}$ are therefore urgently needed, including systems amenable to rapid genetic manipulation and medium to high-throughput screening. The fruit fly, Drosophila melanogaster, has been a valuable tool for studying neurodegenerative disorders, and numerous transgenic models have been reported based on expression of human $A ß$, Tau, and $\alpha$ Syn. In particular, given its accessibility and dispensability, the fly eye has proven a versatile experimental system, and $A ß$, Tau, and $\alpha$ Syn have all been associated with retinal toxicity $[9,20,28$, 33, 52]. However, prior studies of these Drosophila AD/ PD transgenic models have used different expression conditions and assays, hindering systematic comparisons of toxicity profiles. Further, many studies have utilized expression drivers that do not allow differentiation of toxicity in (1) the adult versus developing nervous system and/or (2) neuronal versus non-neuronal supporting cells (glia). Here, we use standardized conditions and assays to systematically examine neurodegenerative changes induced by $A ß$, Tau, and $\alpha$ Syn, including the evaluation of both structural and functional changes following selective expression in the neuronal cells of the adult retina. These studies demonstrate that each protein recapitulates a unique profile of neurodegenerative pathology, demonstrating distinct and apparently separable impacts on neuronal death and dysfunction.

\section{Materials and Methods}

\section{Drosophila Stocks and husbandry}

The following previously reported Drosophila stocks were obtained for our experiments: Rh1-GAL4 (II)[63], UASTau ${ }^{W T 11}$ [62], and UAS-alpha-synuclein [18]. The UAS$\mathrm{Tau}^{\text {WT11 }}$ transgene expresses the full-length, 383-amino acid isoform of human Tau, lacking amino terminal inserts (exons 2 and 3 ) and containing 4 microtubule binding repeats $(0 \mathrm{~N} 4 \mathrm{R})$. The $U A S-A \beta$ and codon-optimized UAS- $\alpha$ Syn strains are newly reported in this study but are both available in the Bloomington Drosophila Stock Center: P\{UAS-APP.Abeta42.B $\} m 26 a$ and P\{UAS-SNCA.J\}7. The UAS-A $\beta$ line consists of the open reading frame encoding the 42 amino acid $A ß$ fragment of human $A P P$ fused with the fly argos secretion signal peptide under the control of UAS in the P\{UAST\} vector. The resulting amino acid sequence is (argos sequence italicized): MPTTLMLLPCMLLLLLTAAAVAVGGDAEFRHDSGYEV HHQKLVFFAEDVGSNKGAIIGLMVGGVVIA

Expression of the $A ß$ peptide was confirmed and quantified by ELISA (Additional file 1: Figure S1). To extract total $\mathrm{A} \beta 42,7$ heads were homogenized in $50 \mu \mathrm{L} \mathrm{GnHCl}$ extraction buffer ( $5 \mathrm{M}$ guanidinium $\mathrm{HCl}$, protease inhibitor cocktail (GenDEPOT) and $50 \mathrm{mM}$ Tris $\mathrm{HCl}, \mathrm{pH}=8.0$ ). Following centrifugation $\left(14,000 \mathrm{~g}\right.$ for $\left.15 \mathrm{~min} @ 4{ }^{\circ} \mathrm{C}\right)$, supernatant was diluted 1:50, 1:100 and 1:200 in standard dilution buffer, before performing ELISA measurements (Invitrogen, KHB3441) following the manufacturer's protocol. The codon-optimized UAS-alpha-synuclein line was developed from a synthetic human alpha-synuclein cDNA engineered by DNA 2.0 (Menlo Park, CA) using codons optimized for insect expression. The resulting cDNA, encoding full-length, wild-type human alpha-synuclein protein, was subcloned into the pExpress-UAS expression vector [43]. Expression of $\alpha$ Syn was confirmed by immunoblot (clone 42, BD Transduction Laboratories, 1:1000) (Additional file 1: Figure S1). Immunoblot was also performed for Tau (Dako, 1:5000). Expression of Tau and $\alpha$ Syn were quantified relative to recombinant, purified protein standards of known concentration (Tau: Abcam, Cambridge, UK; $\alpha$ Syn: EMD Millipore, Temecula, CA). All crosses were established at $18{ }^{\circ} \mathrm{C}$, and adult progeny were transferred to $25^{\circ} \mathrm{C}$ between $0-24$ hours post-eclosion for aging and subsequent analyses. Flies were maintained in continuous, ambient laboratory light conditions. Female flies were used for all analyses.

\section{Retinal histology}

Adult flies were fixed in $8 \%$ glutaraldehyde (EM grade) and embedded in paraffin. Frontal $(5 \mu \mathrm{m})$ and tangential $(3 \mu \mathrm{m})$ retinal sections were prepared on the microtome (Leica) and stained with hematoxylin and eosin. At least 4 animals were examined for each genotype and preparation (frontal and tangential sections). Frontal retinal sections 
(Fig. 2) were photographed at the level of the giant commissure interneurons, posterior to the fan-shaped body. As in prior work [40], we quantified the number of ommatidia in which 7 photoreceptor rhabdomeres remained intact within each field of view (100x) centered within well-oriented, retinal sections from each animal (Fig. 3).

\section{Electrophysiology}

ERG recordings were performed as previously described [64]. In brief, adult flies were anesthetized and glued to a glass slide. A reference electrode was inserted in the thorax and the recording electrode was placed on the corneal surface of the eye. Flies were maintained in the darkness for at least 5 minute prior to a train of 10, 1-second flashes of white light pulses (LED source with daylight filter), during which retinal responses were recorded and analyzed using WinWCP (University of Strathclyde, Glasgow, Scotland) and AxoGraph (Berkeley, CA), respectively. At least 6 flies were examined for each genotype and timepoint.

\section{TEM}

Both retinae and laminae in adult flies were processed for TEM imaging as described [58]. Samples were processed using a Ted Pella Bio Wave microwave oven with vacuum attachment. Adult fly heads were dissected at $25{ }^{\circ} \mathrm{C}$ in $4 \%$ paraformaldehyde, $2 \%$ glutaraldehyde, and $0.1 \mathrm{M}$ sodium cacodylate (pH 7.2). Samples were subsequently fixed at $4{ }^{\circ} \mathrm{C}$ for 48 hours. $1 \%$ osmium tetroxide was used for secondary fixation and subsequently dehydrated in ethanol and propylene oxide, and then embedded in Embed-812 resin (Electron Microscopy Science, Hatfield, PA). $50 \mathrm{~nm}$ ultra-thin sections were obtained with a Leica UC7 microtome and collected on Formvar-coated copper grids (Electron Microscopy Science, Hatfield, PA). Specimens were stained with $1 \%$ uranyl acetate and $2.5 \%$ lead citrate and imaged using a JEOL JEM 1010 transmission electron microscope with an AMT XR-16 mid-mount 16 megapixel CCD camera. For quantification of ultrastructural features, electron micrographs were examined from 3 different animals per genotype.

\section{Statistical analysis}

For primary analyses of ERG (Fig. 1) and rhabdomere loss (Fig. 3), two-way ANOVA was implemented to detect potential differences between group mean outcomes by genotype and age. The overall model F-test is reported in the relevant figure legends. Subsequently, subsetted student's t-tests (2-tailed) were performed for post-hoc comparisons of control animals with each experimental genotype (Tau, $\mathrm{A} ß$, or $\alpha \mathrm{Syn})$, considering each timepoint independently; Bonferroni adjustment was performed in order to correct $\mathrm{p}$-values for multiple comparisons $\left[\mathrm{p}_{\text {adjusted }}=\mathrm{p}_{\text {unadjusted }} * 3\right.$, for $\mathrm{n}=3$ genotypes). For secondary analyses of TEM parameters, statistical comparisons were implemented using a two-tailed student's t-test. Error bars in all analyses represent the standard error of the mean (SEM, $95 \%$ confidence interval).

\section{Results}

The transgenic models utilized for this comparative study take advantage of the GAL4-UAS system [7], in which cDNAs encoding human Tau (0N4R isoform), the Aß42 peptide, or full-length $\alpha$ Syn protein are under control of the heterologous yeast Upstream Activating Sequence $(U A S)$ promoter element and responsive to driver lines expressing the GAL4 transcription factor. We selected the Rhodopsin1-GAL4 (Rh1) line that is expressed following photoreceptor maturation and is thus only active following completion of eye development beginning in late pupal stages [47]. This permits the study of potential toxic properties within neurons, excluding the potential contribution of glial or supporting cells. In addition, use of the $R h 1$ driver further excludes the potential delayed contribution of developmental toxic mechanisms. Since the GAL4-UAS system is temperature sensitive, all crosses were additionally carried out at $18{ }^{\circ} \mathrm{C}$, and shifted to $25^{\circ} \mathrm{C}$ following eclosion, further restricting transgene expression to adulthood [39]. In order to facilitate comparisons of resulting toxicities, we determined the absolute expression level of Tau, Aß, and $\alpha$ Syn under the standardized experimental conditions (Additional file 1: Figure S1). For Tau and $\alpha$ Syn, Western blots prepared from 10-day old adult fly heads and quantified relative to recombinant purified protein standards at known concentration (See Materials and Methods and Additional file 1: Figure S1). Aß was quantified using an established ELISA. Based on this, we estimate the relative molar concentration for expression of Aß, Tau, and $\alpha$ Syn driven by Rh1-Gal4 at 1:36:232, respectively. Animals were examined using complementary structural and functional assays to assess the toxic effects of each human protein in the fly retina. For structural studies, we used hematoxylin and eosin stained histologic sections to reveal photoreceptor integrity in the retina and lamina. Retinal function was probed using the ERG, permitting assessment of light-induced photoreceptor field potentials and efficacy of synaptic transmission [60].

\section{Tau}

In prior work, expression of human Tau transgenes in the fly nervous system has been associated with substantial neurotoxicity, including in the fly eye, and these models have proven useful for both mechanistic investigation and genetic screening [19]. The most widely deployed assay has been the "rough eye", typically induced using the GMR-GAL4 driver, in which disrupted retinal development leads to a reduced eye size and a roughened surface. While the Tau-induced rough eye has shown great utility for genetic studies, especially modifier screens [1, 5, 52], 


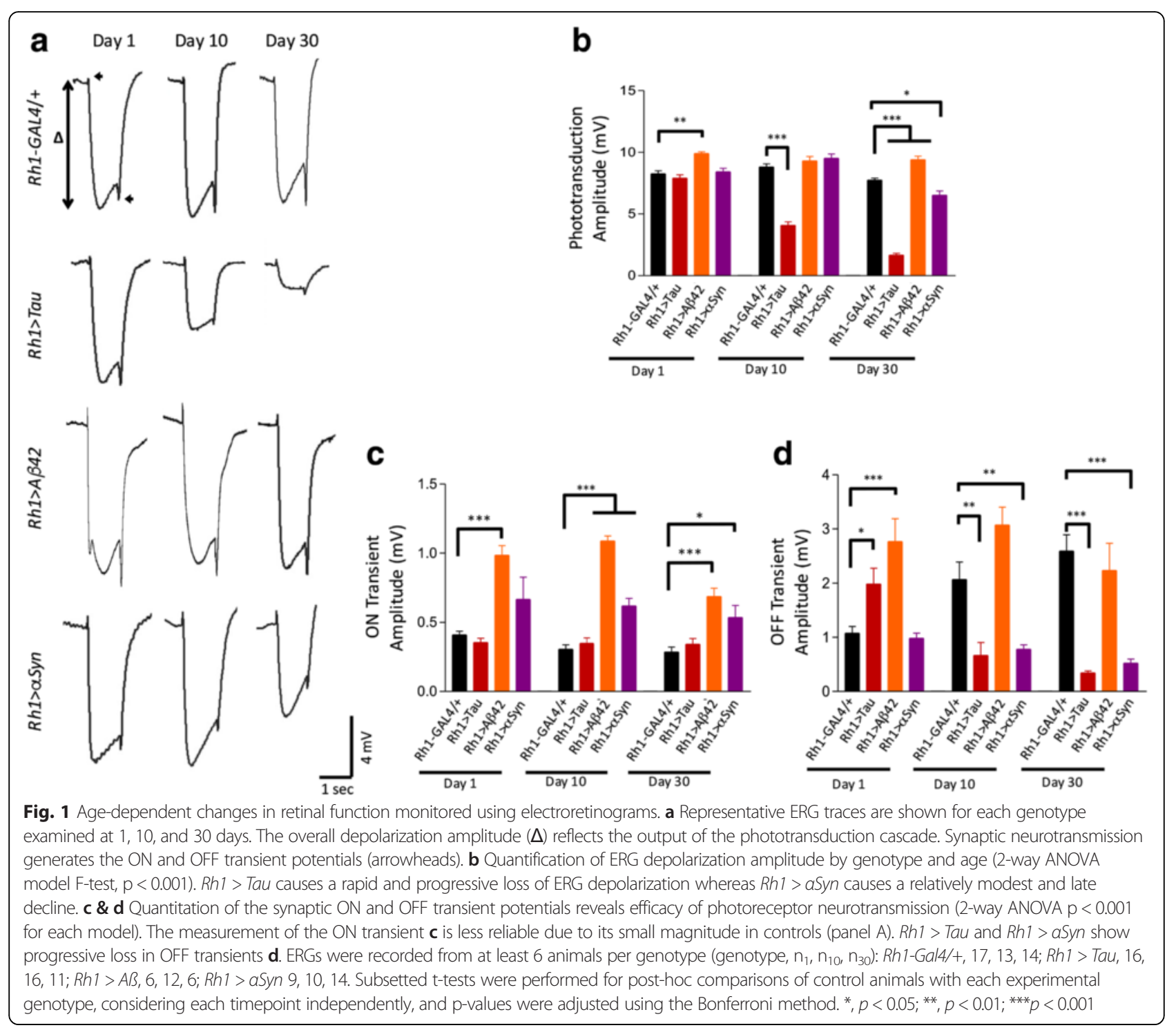

potential limitations include the developmental component, as well as an inability to examine age-dependent changes, since the phenotype is established during development. Previously published and well-characterized UAS:Tau transgenic lines [62], expressing the 383 amino acid, wild-type human 0N4R isoform, were crossed to the $R h 1$ driver. Compared to the rough eye caused by other eye drivers, the external eye appearance of $R h 1>\mathrm{Tau}$ is indistinguishable from control animals (Additional file 2: Figure S2).

ERGs permit assessment of light induced field potentials serving as a sensitive indicator of photoreceptor function, retinal integrity/degeneration, and efficacy of synaptic transmission [60]. On eclosion (1 day old), Rh1 > Tau flies demonstrated normal ERGs, consisting of an approximately $10 \mathrm{mV}$, light induced depolarization (Fig. 1). However, with aging, Tau expression was associated with a significant loss of the light-evoked ERG response, consistent with progressive photoreceptor dysfunction. By contrast with preserved ERGs in aged Rh1-GAL4 flies, Rh1> Tau showed a $51 \%$ reduction $(p<0.001)$ in phototransduction (depolarization amplitude) by 10 days progressing to an $81 \%$ loss $(p<$ 0.001 ) by 30 days (Fig. 1). We also quantified changes in the ERG ON/OFF transient responses, which denote synaptic neurotransmission. Under the conditions used for ERG recordings, it was difficult to detect robust reductions in the ON transient potential due to its small magnitude ( $\sim .5 \mathrm{mV}$ in Rh1-GAL4 / + controls). However, at all three time points examined, we observed significant reductions in the ERG OFF transient potential compared to controls (Fig. 1d).

In order to assess the integrity of the Drosophila visual system, and to examine for photoreceptor cell loss, we prepared semi-thin sections of fixed and paraffin-embedded 
heads, staining with hematoxylin and eosin. Frontal (Fig. 2) and tangential (Fig. 3 and Additional file 3: Figure S3) microtome sections provide complementary views of retinal structure, allowing detection of any vacuolar changes and tissue architectural disruption. Tangential sections through photoreceptors readily permit sensitive detection of photoreceptor cell loss through counts of rhabdomeres, specialized organelles that mediate phototransduction. In contrast to the rapidly progressive loss of ERG responses, we did not observe any significant histologic changes in the retina in $R h 1>$ Tau flies; numbers and morphology of photoreceptors were preserved at all timepoints (Figs. 2 \& 3). At 30 days, $92 \%$ of ommatidia in quantified sections from Rh1 > Tau retinae demonstrated intact ommatidia with 7 visible rhabdomeres at the level examined, which was not significantly different from Rh1-GAL4/+ controls (Fig. 3). Thus, when expressed in adult photoreceptors, human Tau induces progressive neuronal dysfunction, independent of any detectable neuronal loss or obvious retinal tissue changes.

\section{Aß42}

Similar to Tau, Aß expression has previously been demonstrated to be toxic to Drosophila eye tissue, causing both disrupted architecture and a rough eye phenotype [10, 21]. These studies have also predominantly relied on the GMRGAL4 expression driver, which expresses throughout development, including both photoreceptors and supporting, non-neuronal cells, and produces a rough eye. When expression of the 42-amino acid $A ß$ peptide (Aß42, referred to hereafter as $A ß)$ is restricted to adult, differentiated photoreceptors, using $R h 1>A \beta$, animals have wildtype appearing eyes upon visual inspection, without a detectable roughening (Additional file 2: Figure S2). In contrast to $R h 1>T a u$, ERG recordings from $R h 1>A \beta$ animals do not show any evidence of rapidly progressive loss in neuronal function (Fig. 1). In fact, both photoreceptor depolarization and the associated transient responses were significantly increased in $R h 1>A \beta$ flies at multiple timepoints. On examination of retina histology, 1-day old animals showed normal appearing retinal tissue morphology and architecture (Fig. 2), and a full complement of photoreceptors was observed in each ommatidium on tangential sections (Fig. 3). Upon aging, however, we observed progressive vacuolar changes affecting the retina, similar to those previously reported in $G M R>A \beta$ flies [21]. In addition, by 30 days we detected significant reductions in rhabdomere numbers $(p<0.001)$ consistent with neuronal loss, such that only $57 \%$ of ommatidia in retinae from $R h 1>A \beta$ flies were intact, whereas similar changes were not seen in Rh1 > Tau (Fig. 3b). Thus, compared with Tau, Aß induces a markedly distinct pattern of retinal neurodegeneration, consisting of progressive structural degeneration and neuronal loss with overall preserved photoreceptor functioning.

\section{aSyn}

Unlike $A ß$ and Tau, $\alpha$ Syn expression in the Drosophila eye using the GMR-GAL4 driver line does not produce a rough-eye phenotype. However, modest evidence of toxicity has been reported on retinal histology [2, 18], including

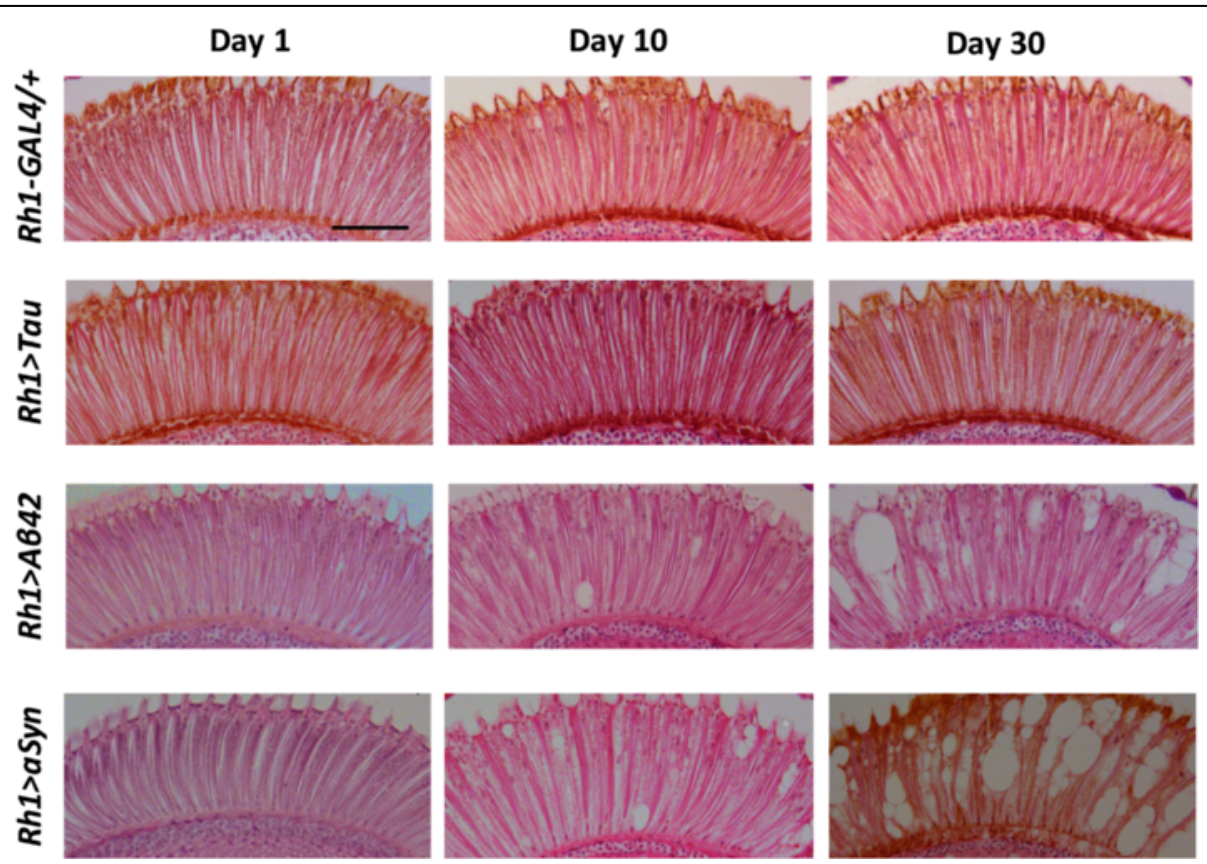

Fig. 2 Representative frontal sections of the Drosophila retina stained with hematoxylin and eosin demonstrate age-dependent vacuolar change and tissue loss in $R h 1>A B$ and $R h 1>a S y n$, but relative preservation $R h 1>$ Tau. Scale bar: $50 \mu m$ 


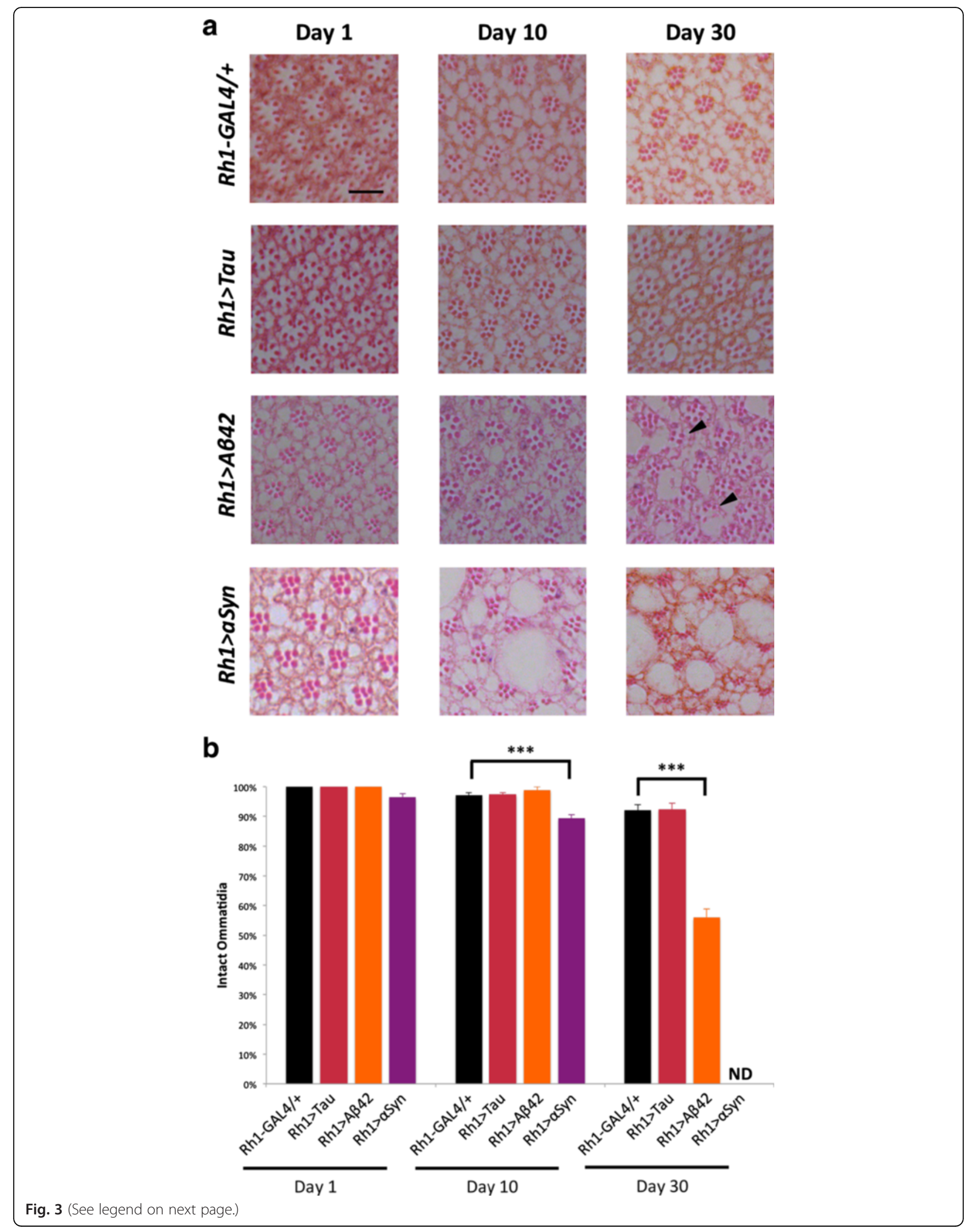


(See figure on previous page.)

Fig. 3 a Representative tangential sections of Drosophila retina stained with hematoxylin and eosin show progressive neuronal loss in $R h 1>A B$ and Rh1 > aSyn, but not in Rh1 > Tau. Arrowheads highlight representative ommatidia with $<7$ rhabdomeres. Scale bar: $10 \mu m$. See also Additional file 3: Figure S3 for low-power views. $\mathbf{b}$ Photoreceptor loss in tangential sections was quantified based on the percentage of ommatidia with a preserved complement of 7 rhabdomeres at each timepoint (2-way ANOVA model F-test, $p<0.001$ ). In 30 day-old animals, only $56 \%$ of ommatidia in retinae from $R h 1>A B$ flies had the full complement of 7 rhabdomeres, compared with $92 \%$ in Rh1-GAL4/+ controls ( $p<0.001)$, whereas no significant change was seen in Rh1 > Tau (92 \%). Given the severity of tissue loss at 30 days, it was not possible to identify preserved ommatidia in Rh1 > aSyn flies (ND); however, in 10 day-old animals, $89 \%$ of ommatidia were intact, compared to $97 \%$ of controls $(p<0.001)$, consistent with photoreceptor loss. Approximately 100 ommatidia were examined per 100x field of view for at least 4 animals per genotype. Total number of retinae examined (genotype, $n_{1}, n_{10}, n_{30}$ ): Rh1-Gal4/t, 9, 8, 7; $R h 1>$ Tau, 7, 7, 10; Rh1 >AB, 7, 8, 8; Rh1 >aSyn 7, 7, ND. Subsetted t-tests were performed for post-hoc comparisons of control animals with each experimental genotype, considering each timepoint independently, and p-values were adjusted using the Bonferroni method. At Day 1 , SEM $=0$ for Rh1-Gal4/t, Rh1 $>$ Tau, and Rh1 $>A$ A . ***, $p<0.001$

vacuolar changes, and one study reported ERG changes potentially consistent with disrupted neurotransmission [25]. When previously published UAS: $\alpha$ Syn lines [18] were expressed using the $R h 1$ driver, we did not detect any appreciable changes in the ERG depolarization, including with aging, and only minimal changes were detected on retinal histology (Additional file 4: Figure S4). Based on the discovery of rare familial forms of synucleinopathy caused by $S N C A$ locus multiplication [53], $\alpha$ Syn neurotoxicity is dosesensitive, and this conclusion has also been supported by studies of mammalian models [16]. We therefore sought to develop and test new $\alpha$ Syn transgenic lines, in which codon usage in the full-length human $\alpha$ Syn cDNA was optimized for Drosophila, permitting more efficient protein translation. Indeed, based on western blot analysis, these lines demonstrate significantly increased $\alpha$ Syn expression levels (Additional file 4: Figure S4). Consistent with this observation and compared to $\alpha$ Syn transgenic flies without codonoptimization, files expressing codon-optimized $\alpha$ Syn under control of the Rh1-GAL4 driver (hereafter referred to as $R h 1>\alpha S y n)$ demonstrated a markedly enhanced toxicity profile. Using the ERG assay, we find that depolarization amplitude is initially preserved, but exhibits a modest but significant, age-dependent decline (versus $R h 1 /+$ controls) which is apparent at 30 days $(8 \%$ reduction, $\mathrm{p}<0.05)$ (Fig. 1b). ERG off transient potentials show a consistent, age-dependent loss in $R h 1>\alpha$ Syn flies (Fig. 1d). On histological analysis, the retinae of $R h 1>\alpha S y n$ flies initially display no obvious morphological defects, but aging leads to progressive vacuolar changes (Fig. 2) and rhabdomere loss (Fig. 3), ultimately leading to substantial architectural disruption and tissue destruction. In 10 day-old animals, we detected modest but significant reductions in rhabdomere numbers $(\mathrm{p}<0.001)$ consistent with neuronal loss, such that $89 \%$ of ommatidia in retinae from $R h 1>\alpha$ Syn flies were intact, compared to $97 \%$ in controls (Fig. 3b). By 30 days, the severity of tissue loss and architectural distortion made it impossible to identify preserved ommatidia. In sum, $\alpha$ Syn expression shows a third characteristic profile of neurodegeneration in the Drosophila visual system distinct from either Tau or Aß, causing substantial age-dependent structural degenerative changes, including cell death, with comparatively delayed, modest functional deficit based on ERG depolarization.

\section{Transmission electron microscopy}

In order to reveal potential mechanisms underlying the distinct patterns of toxicity seen using medium throughput assays, and to understand the discordance between structural and functional assays, we performed TEM to examine photoreceptor ultrastructure following expression of Aß, Tau, or $\alpha$ Syn with the Rh1-GAL4 driver. We used identical conditions to those described above, and performed our analysis at 10 days in order to capture the evolution of age-dependent changes. Based on retinal TEM, all three toxic proteins induced modest disruptions in photoreceptor rhabdomeres (Fig. 4), specialized membranerich, electron dense organelles that mediate phototransduction and thereby generate the ERG depolarization. Compared to $R h 1 /$ + controls, expression of Tau, Aß, or $\alpha$ Syn caused subtle defects in rhabdomere architecture, including apparent splitting / "fraying" at the edges, vacuolar changes, and other irregularities. However, the frequency of these changes did not appear to correlate well with the extent and timing of functional deficits as revealed by ERG. Consistent with findings on retinal histology, rhabdomere disruption was most frequent in $R h 1>\alpha \operatorname{Syn}(\sim 65 \%$, Fig. 4e) even though significant changes in ERG depolarization are not observed at this timepoint. Conversely, despite the profound reduction in the ERG response characteristic of $R h 1>\mathrm{Tau}$ flies at 10 days (Fig. 1), the majority of photoreceptors had preserved rhabdomeres. The resolution afforded by TEM also allowed us to examine several subcellular systems implicated in neurodegeneration (Fig. 5). While mitochondrial numbers and morphology were preserved (Fig. 5e), we observed evidence of increased endolysosomal-dependent processes (Fig. $5 \mathrm{f} \& \mathrm{~g}$ ). Specifically, in both $R h 1>A \beta$ and $R h 1>\alpha$ Syn, we see increased numbers of multivesicular or multilamellar bodies, consistent with altered autophagic flux, whereas in $R h 1>T a u$, a preponderance of electron dense vesicles were seen consistent with the appearance of telolysosomes (also called residual bodies or lipofuscin granules). Electron-dense material, possibly consistent with granular 

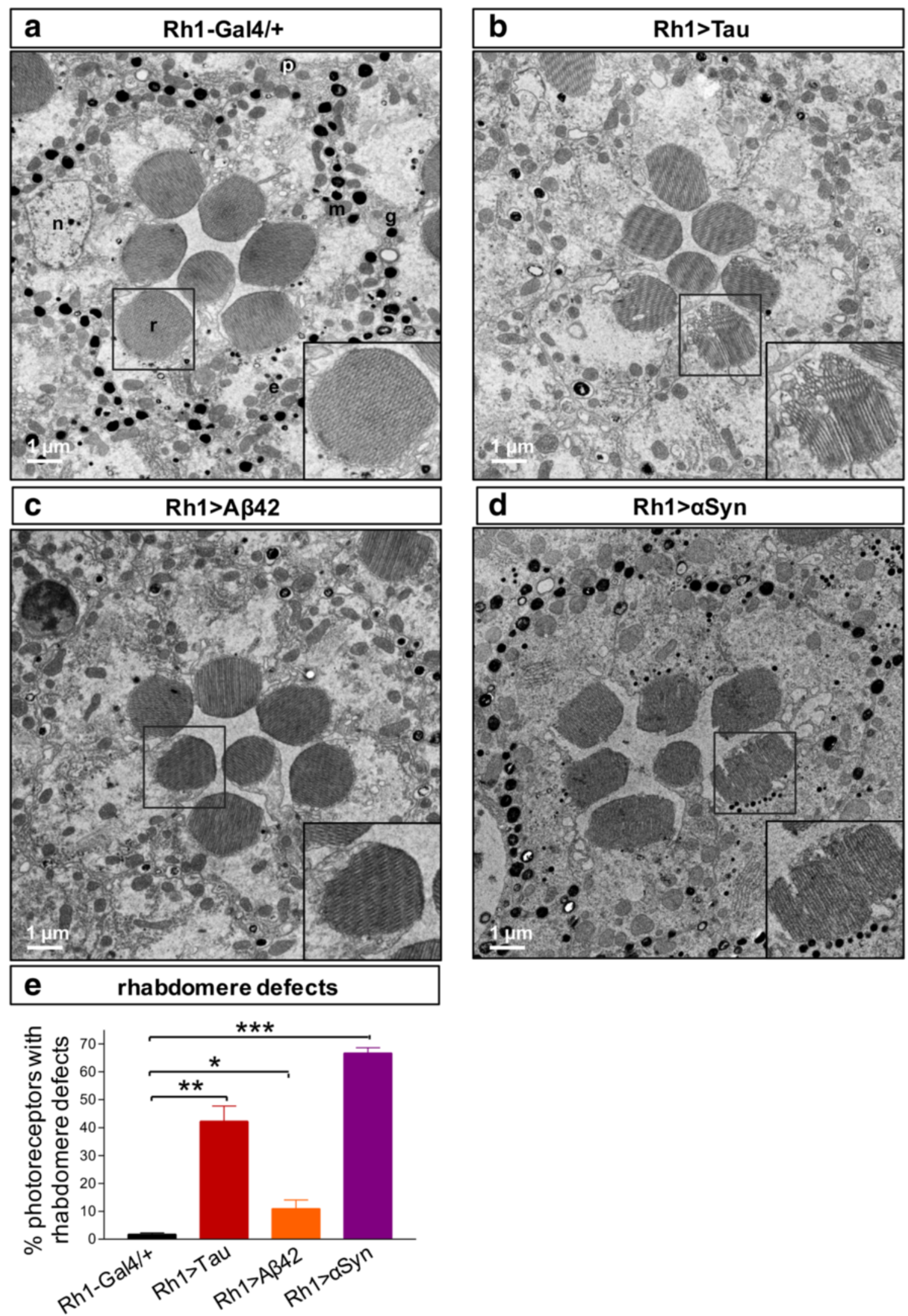

Fig. 4 (See legend on next page.) 
(See figure on previous page.)

Fig. 4 Compared to controls (a, Rh1-GAL4 / +), TEM reveals modest ultrastructural defects in rhabdomeres of $R h 1>\operatorname{Tau} \mathbf{b}, R h 1>A B \mathbf{c}$ and $R h 1>$ aSyn $\mathbf{d}$ flies at 10 days. a Photoreceptor subcellular organelles are labelled, including nucleus ( $\mathrm{n}$ ), rhabdomere (r), mitochondria (m), and rough endoplasmic reticulum (e). Non-neuronal pigment (p) cells and glia (g) are also noted. e Frequency of photoreceptors with rhabdomere structural defects was quantified from electron micrographs taken from 3 distinct animals per genotype. Total number photoreceptors quantified (genotype, n): $R h 1-G A L 4 /+, 614 ; R h 1>A B, 326 ; R h 1>$ Tau, 389; and $R h 1>a S y n, 330$. Scale bar: $1 \mu$ m. Statistical comparisons were implemented using a two-tailed student's t-test. ${ }^{*}, p<0.05 ;{ }^{* *}, p<0.01 ;{ }^{* *}, p<0.001$

tau oligomeric aggregates, has previously been observed in Tau transgenic flies [15].

We next examined the organization and morphology of photoreceptor synaptic terminals, based on TEM of the lamina. In control animals $(R h 1 /+)$, TEM reveals a regular array of "cartridges" in which 6 photoreceptor synaptic terminals converge and surround the centrally located dendritic projections from second order neurons in the Drosophila visual system (Fig. 6a). The cytoplasm of the photoreceptor terminals appears electron dense relative to the dendritic processes due to the synaptic vesicular contents. Expression of Tau or $\alpha$ Syn, but not Aß, significantly disrupted photoreceptor synaptic terminal organization and/or morphology. Remarkably, in Rh1>Tau animals examined at 10 days, it was no longer possible to discriminate photoreceptor terminals (Fig. 6a), perhaps due to a paucity of synaptic vesicles, and the overall architecture of the lamina was disturbed. By contrast, in $R h 1>\alpha S y n$, overall lamina organization was preserved; however, synaptic terminals were significantly enlarged, assuming a bulbous appearance (Fig. 6d and e), and synaptic vesicle density was found to be decreased (Fig. 6f). Additionally, $\alpha$ Syn expression appeared to alter synaptic vesicle size, leading to enlarged and more heterogeneous vesicles (inset, Fig. 6d). In sum, TEM reveals significant but distinct patterns of synaptotoxicity following expression of Tau and $\alpha$ Syn in adult photoreceptors.

\section{Discussion}

We report on parallel, systematic assessments of both structural and functional changes occurring in the Drosophila retina following expression of human Tau, Aß, and $\alpha$ Syn under identical conditions. Remarkably, these toxic protein species are discovered to recapitulate distinct profiles of neurodegeneration, and the findings highlight a surprising "uncoupling" between neuronal dysfunction and retinal destruction/cell loss. Expression of Tau in the adult retina causes a significant and progressive decline in photoreceptor function, based on the ERG, whereas tissue histology reveals preserved retinal architecture without evidence of structural degeneration. As discussed further below, Taurelated neuronal dysfunction has been reported in several prior Drosophila studies using a variety of complementary expression conditions and assays [12, 36, 38]. By contrast, we show that $A ß$ expression has minimal impact on the ERG but causes progressive retinal vacuolar changes and associated rhabdomere loss. $\alpha$ Syn expression-based on new, codon-optimized transgenes-generates a third profile consisting of rapid, age-dependent histologic destruction of retinal tissue with comparatively late, and modest change in photoreceptor field potentials. Finally, ultrastructural analyses reveal evidence of altered endolysosomal pathways following expression of all three proteins, and in the case of Tau and $\alpha$ Syn, synaptotoxic mechanisms are strongly implicated. Interestingly, mitochondrial numbers and morphology were preserved in all 3 models. The assays employed in our studies (histology, ERG, and TEM) are highly complementary, allowing assessments of neurodegenerative changes at the macro-, micro-, and ultra-structural levels as well as correlation with retinal neurophysiology. Based on numerous prior studies, the mechanisms of Aß-, Tau-, and $\alpha$ Syntriggered neuronal injury in $\mathrm{AD}$ and $\mathrm{PD}$, respectively, have many features in common, including aggregation, pathologic propagation [59], and overlapping cytotoxic profiles (e.g. synaptic changes [4, 56], mitochondrial dysfunction/oxidative stress [24, 61], and altered proteostasis [34]). Despite these parallels, the distinct degenerative phenotypes observed following expression of Tau, Aß, and $\alpha$ Syn expression in the Drosophila eye suggest these protein species engage at least partially non-overlapping downstream effector mechanisms.

While we have taken several steps to standardize expression conditions for $\mathrm{Tau}, \mathrm{A} \beta$, and $\alpha \mathrm{Syn}$, it is not possible to exclude the contribution of variation in transgene/protein expression levels to the observed toxicity profiles. Indeed, the neurotoxicity of these protein species is highly dosedependent in most experimental systems, and human genetic variants that increase $A \beta$ and $\alpha$ Syn production are risk factors for $\mathrm{AD}$ and $\mathrm{PD}$, respectively [32, 46]. Based on quantitative Western blots and ELISA, we estimated the relative expression of $A \beta$, Tau, and $\alpha$ Syn under our experimental conditions was approximately 1:30:200 (by molarity), respectively (Additional file 1: Figure S1). The comparatively high $\alpha$ Syn expression levels likely relates to codon-optimization, which achieves $\sim 20$-fold higher expression compared to previously published transgenic strains (Additional file 4: Figure S4). While we estimate that non-codon optimized $\alpha$ Syn has more comparable expression levels (Aß:Tau: $\alpha$ Syn $=1: 30: 10$ ), this line demonstrates minimal toxicity in our assays, perhaps consistent with more robust neuronal compensatory mechanisms. 


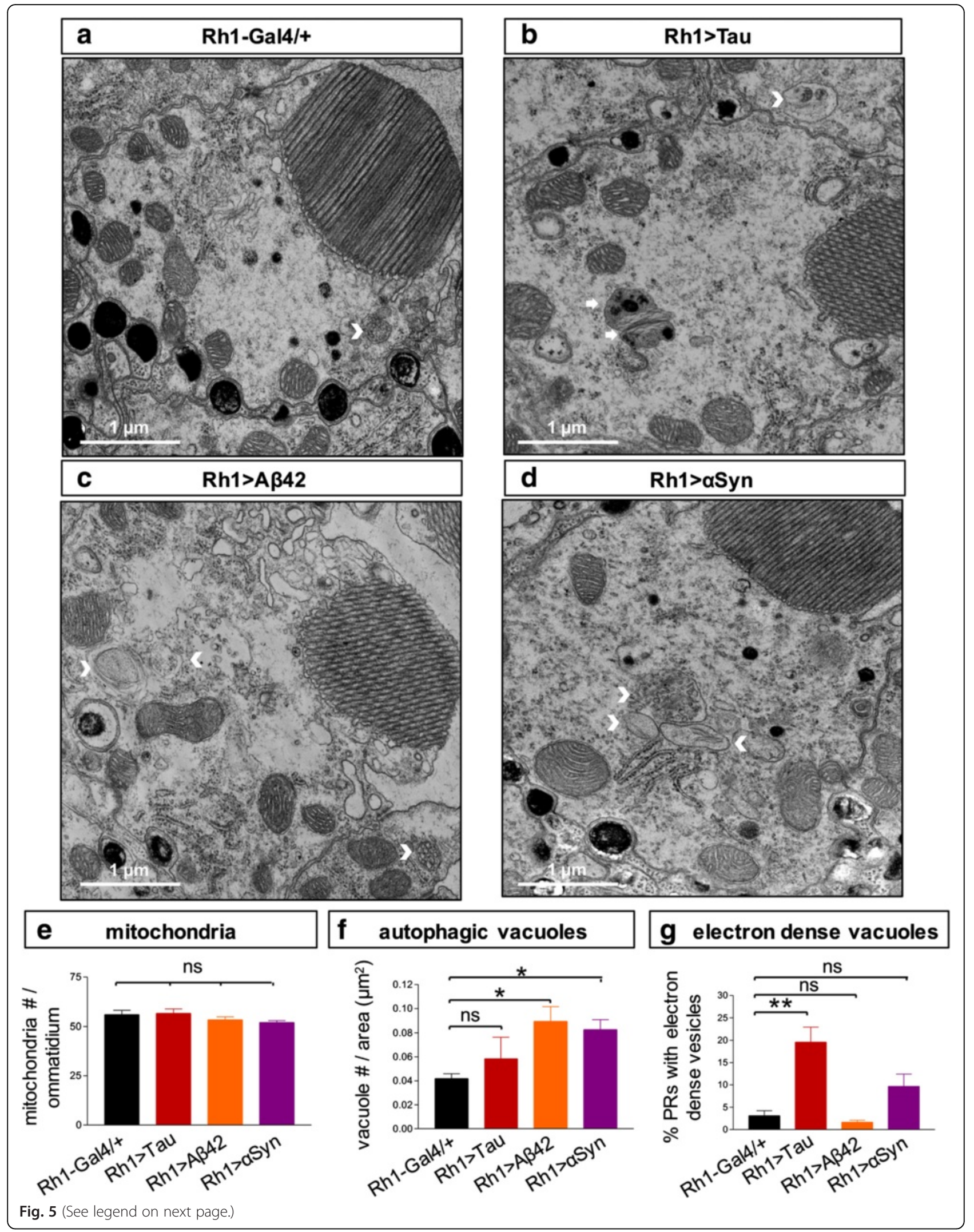




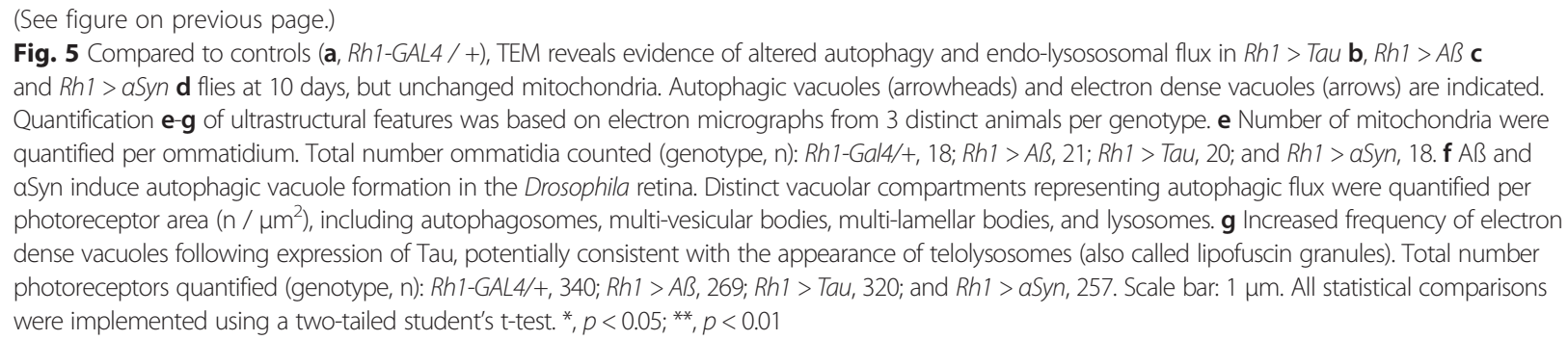

Nevertheless, variation in protein expression levels seem unlikely to account for many of our observations, since higher expression was in fact a poor predictor of enhanced toxicity. For example, despite its relatively higher expression over Tau, $\alpha$ Syn caused a comparatively modest and late disruption in the ERG. Moreover, despite the significantly lower expression, $A ß$ but not Tau triggered retinal vacuolar changes and rhabdomere loss. In the future, it may be informative to repeat our studies following codon-optimization of $A ß 42$ and Tau and/or with transgenic strains that use the same genomic insertion site.

One strength of our study is the choice of expression conditions facilitating comparison of toxicity profiles for Tau, $A \beta$, and $\alpha$ Syn in adult neurons. We selected the Rh1-GAL4 expression driver which is activated shortly prior to eclosion of adult flies within mature, fully differentiated photoreceptors and following patterning and morphogenesis of the eye $[37,47,48,51]$. While the spatiotemporal expression profile of the $R h 1$ promoter has been extensively-characterized, we cannot exclude the possibility of low-level "leaky" transgene expression. In order to further suppress expression during development, including late pupal stages, crosses were established at $18{ }^{\circ} \mathrm{C}$ and adult animals were shifted to $25{ }^{\circ} \mathrm{C}$ following eclosion. Most prior work using retinal phenotypes for fly neurodegenerative models have relied on drivers that express during eye development (GMR-, $g l$-, and Sev-GAL4). When Aß [21] or Tau [62] is expressed in this manner, retinal morphogenesis is disrupted resulting in a "rough eye". Importantly, the rough eye phenotype is readily amenable to enhancer/suppressor screening, and successful large-scale and unbiased modifier studies have been performed in both Tau $[1,5,52]$ and $A ß$ [9] transgenic flies. However, these developmental driver lines make it difficult to study the toxicity of Tau in mature neurons, potentially most relevant for adult-onset neurodegenerative disorders such as $\mathrm{AD}$ and PD. Notably, the ERG assay used here has also been deployed successfully for large-scale genetic screens [64]. Another potential advantage of $R h 1$ is its selectivity for retinal photoreceptors compared to GMR-GAL4, which is expressed more broadly within both neuronal and supporting cells, including glia. Based on studies in the brain, Tau can also recapitulate toxicity in Drosophila glia, and neuron-glia interactions can influence overall levels of degeneration in the nervous system [14]. In sum, in contrast to much prior work using the Drosophila eye as a tissue for the study of neurodegeneration, our findings selectively highlight the impact of $A ß$, Tau, or $\alpha$ Syn in post-mitotic neurons.

One unexpected finding is the progressive photoreceptor dysfunction in $R h 1>$ Tau flies that occurs in the absence of apparent retinal histologic degeneration or neuronal death. This contrasts not only with the effects of $A ß$ or $\alpha$ Syn when expressed under identical conditions, but also with the profound rough eye and associated retinal histologic changes following developmental expression of Tau (e.g. GMR-GAL4). Even with the increased resolution provided by TEM, retinal architecture was surprisingly preserved with significant loss of photoreceptor depolarization out of proportion to changes in the responsible cellular compartment/structures (i.e. ommatidia/ rhabdomeres). Notably, prior studies of Tau-induced progressive neurodegeneration in the adult brain [62] have relied on drivers, such as elav-GAL4, which are also expressed early in neuronal differentiation. Overall brain morphology is preserved in these flies; although aberrant development of selected neuronal structures, such as the mushroom bodies, have been documented [30]. Potentially related to our observations of "uncoupling" between neuronal dysfunction and degeneration, Kosmidis et al. found that certain mutant forms of Tau expressed under the control of elav-GAL4 disrupt Drosophila olfactory memory, while apparently preserving mushroom body morphology [30]. In the context of glia, Colodner and Feany demonstrated that conditional expression of wildtype Tau in the adult fly brain is capable of inducing progressive apoptotic degeneration [14]. Under conditions in which neuronal expression of Tau is restricted to the adult brain, Papanikolopoulou and Skoulakis showed that flies have decreased survival, but neuronal loss and/or vacuolar changes was not examined [45]. In studies of the larval peripheral nervous system, Mudher et al. found that Tau is capable of disrupting axonal transport [38], and Chee et al. documented Tau-induced synaptic dysfunction [12], both in the absence of apparent neuronal death. Our findings of preserved photoreceptors in the retinae of 

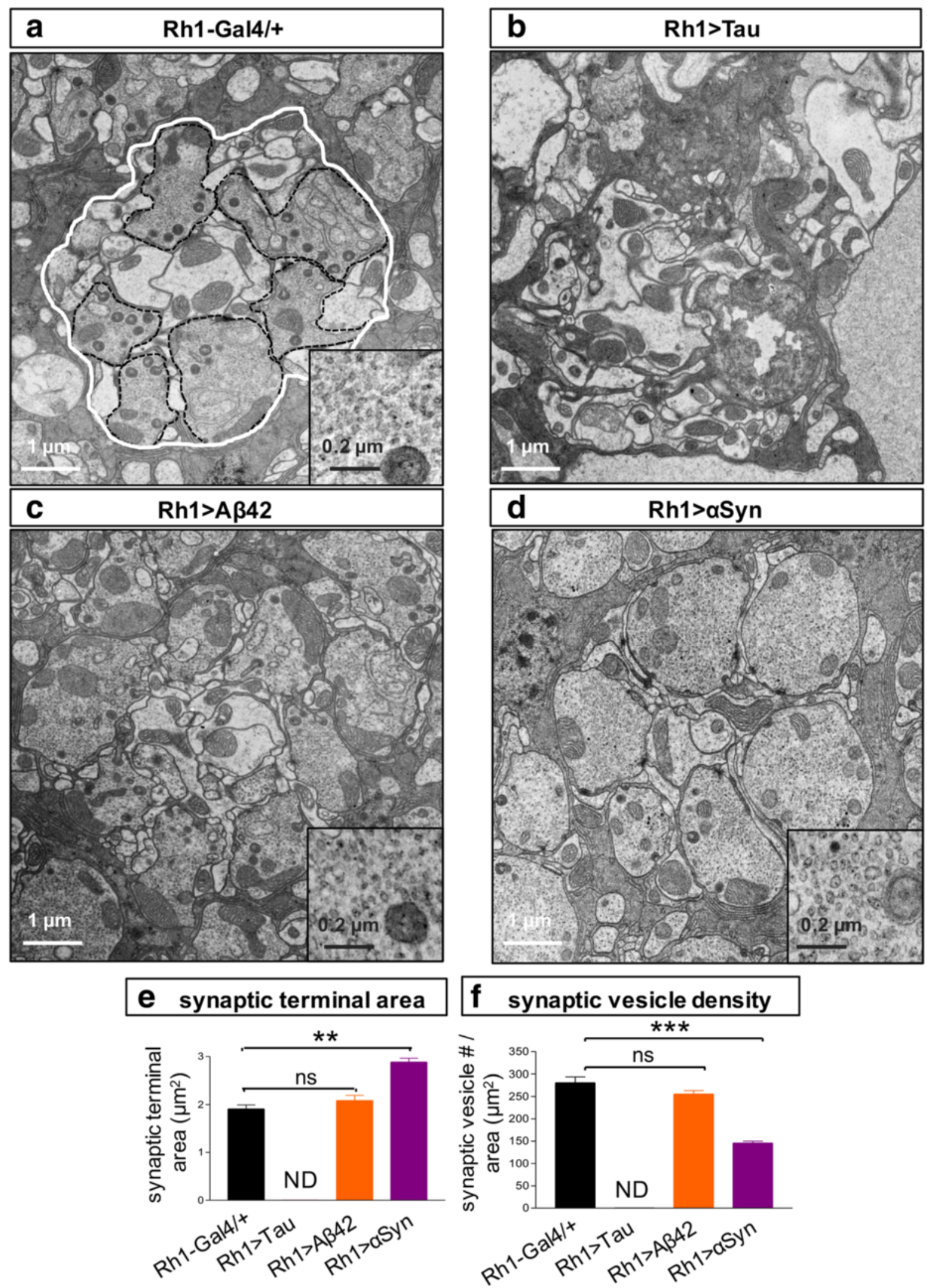

Fig. 6 (See legend on next page.) 


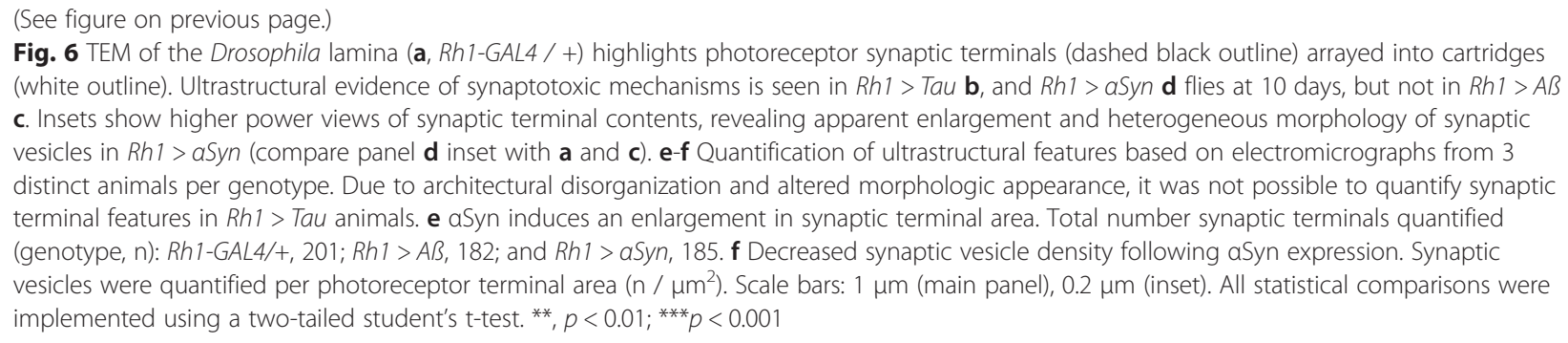

Rh1 > Tau flies appears contradictory to recent findings by Gorsky et al. [22]; however, our studies differ in the specific Tau isoform examined, expression driver/conditions, and assays employed. Tau phosphorylation is also likely an important determinant of the specific profile of Tau toxicity, based on numerous studies in Drosophila [1, $11,42,45,57]$ and many other experimental systems [23]. In sum, the alternative outcomes of neuronal dysfunction versus death following Tau expression may be sensitive to many factors, including timing, cell-type, protein levels, isoform, and post-translational modifications.

Early synaptotoxic mechanisms are emerging as important in the pathogenesis of both $\mathrm{AD}$ and $\mathrm{PD}[44,49,56]$. Based on TEM, we observed profound disruption of photoreceptor terminals in both $R h 1>T a u$ and $R h 1>\alpha S y n$ flies; although, the character of the specific defect was distinct. Tau was associated with a disorganized lamina architecture, and it was no longer possible to differentiate photoreceptor terminals. This is consistent with prior findings of reduced synaptic markers in Tau flies out of proportion to neuronal cell loss [35]. One attractive hypothesis is that these changes might arise from Tau-mediated disruption of axonal transport, leading to impaired delivery of mitochondria and synaptic vesicles and resulting in terminal collapse, consistent with prior observations in a variety of Tauopathy models $[17,26$, 38]. Following $\alpha$ Syn expression, TEM revealed an expansion of the synaptic terminals and enlarged but decreased density of synaptic vesicles. Notably, similar changes have been documented in the brains of $\alpha$ Syn transgenic mice $[6,41,50]$. $\alpha$ Syn avidly binds cellular membranes, including synaptic vesicles and other endomembrane organelles, likely accounting for its conserved activity to remodel and disrupt such systems[4]. Consistent with our TEM findings of disrupted synaptic ultrastructure, Tau and $\alpha$ Syn were each additionally associated with progressive decline in the ERG OFF transient potentials, suggesting impaired neurotransmission [60]. Despite the early and potent synaptotoxicity associated with $A ß$ in many other systems $[44,56]$, we did not detect significant disruptions in the lamina ultrastructure of $R h 1>A \beta$ flies. It will be important in the future to examine for possible emergence of synaptic or other ultrastructural changes at later timepoints since the $\mathrm{A} ß$-induced histologic findings are quite sparse at 10 days and could potentially be missed by TEM due to sampling. Interestingly, despite the age- dependent photoreceptor loss triggered by $A ß$, we observed an initial increase in both the ERG ON/OFF transients along with the overall increased depolarization amplitude. One speculative possibility is that these findings reflect cellular and synaptic functional compensatory mechanisms. While additional studies will be required to understand these dynamic neurophysiologic changes, we note that apparently paradoxical effects have been previously reported for $A B$ species at Drosophila neuromuscular junctions and in mammalian neurons $[13,65]$.

\section{Conclusions}

Based on a combination of functional neuroimaging and clinicopathologic correlation studies, there is increasing recognition that synaptic and circuit dysfunction contribute significantly to $\mathrm{AD}$ and $\mathrm{PD}$ manifestations, and may in fact precede neuronal death and tissue atrophy [27, 29]. It will be important to directly address this experimentally in model systems, and to develop assays that allow mechanistic studies of functional degenerative changes. In Drosophila, $\mathrm{A} \beta, \mathrm{Tau}$, and $\alpha$ Syn transgenic models have been previously utilized for electrophysiological studies at the larval neuromuscular junction $[8,12,13]$, but this approach is potentially limited for the analysis of age-dependent progressive changes in adult neurons. Nervous system function has also been probed in fly disease models based on learning and locomotion in adult animals [19]; however, as discussed above, the relative contribution of neuronal loss versus dysfunction to these behaviors remains to be carefully elucidated. In sum, the parallel studies of retinal ERG, histology, and TEM reported here provide a foundation for future genetic dissection of mechanisms underlying progressive synaptic and functional disruption in transgenic models of $\mathrm{AD}$ and $\mathrm{PD}$, and its relation to the cumulative and potentially irreversible burden from tissue destruction and neuronal loss.

\section{Additional files}

Additional file 1: Figure S1. Quantitation of transgenic expression of Tau, A $\beta$, and aSyn. (PDF $130 \mathrm{~kb}$ )

Additional file 2: Figure S2. External eye appearance of genotypes evaluated in this study. (PDF $177 \mathrm{~kb}$ )

Additional file 3: Figure S3. Low power histologic sections. (PDF 158 kb) 
Additional file 4: Figure S4. Expression and toxicity of non-codon optimized aSyn. (PDF $141 \mathrm{~kb}$ )

\section{Competing interest}

The authors declare that they have no conflict of interest.

\section{Authors' contributions}

AKC, CG, Y-CH, HY and MS contributed to study design, performed the experiments, interpreted/analyzed the data, and participated in drafting and critical revision of the manuscript. $\mathrm{ZZ}$ and $\mathrm{YL}$ performed experiments, collected data and participated in critical revision of the manuscript. SC, JB, and GRJ developed key reagents and participated in critical revision of the manuscript. GRJ and JB additionally funded portions of the study. HJB and JMS contributed to overall study design, acquisition of funding, interpreting the data and critical revisions of the manuscript. JMS additionally prepared the initial manuscript draft. All authors read and approved the final manuscript.

\section{Acknowledgements}

We thank our colleague, Mel Feany, and the Bloomington Drosophila stock center for provision of fly reagents. We also thank our colleagues, Alexandra Litvinchuk and Hui Zheng for assistance with the Aß ELISA. J.M.S. was supported by grants from the NIH (K08AG034290, R21NS089854, R01AG033193, U01AG046161, R01AG050631, C06RR029965), the Alzheimer's Association, the American Federation for Aging Research, Huffington Foundation, the Robert and Renee Belfer Family Foundation, the Jan and Dan Duncan Neurological Research Institute at Texas Children's Hospital, and a Career Award for Medical Scientists from the Burroughs Wellcome Fund. The codon-optimized synuclein flies were developed using support from the NIH (P01ES16732 to GRJ). The Pathology and Histology Core at Baylor College of Medicine is supported by NIH grant P30CA125123. The work was additionally supported by U54HD083092 from the Eunice Kennedy Shriver National Institute of Child Health \& Human Development.

\section{Author details}

${ }^{1}$ Department of Neurology, Baylor College of Medicine, Houston, TX 77030, USA. ${ }^{2}$ Department of Neuroscience, Baylor College of Medicine, Houston, TX 77030, USA. ${ }^{3}$ Department of Molecular and Human Genetics, Baylor College of Medicine, Houston, TX 77030, USA. ${ }^{4}$ Program in Developmental Biology, Baylor College of Medicine, Houston, TX 77030, USA. ${ }^{5}$ Department of Neurology, University of Texas Medical Branch, Galveston, TX 77555, USA. ${ }^{6}$ Parkinson's Disease Research, Education, and Clinical Center, Michael E. DeBakey VA Medical Center, Houston, TX 77030, USA. ${ }^{7}$ Howard Hughes Medical Institute, Baylor College of Medicine, Houston, TX 77030, USA. ${ }^{8}$ Jan and Dan Duncan Neurological Research Institute, Texas Children's Hospital, 1250 Moursund Street, Suite N.1150, Houston, TX 77030, USA

Received: 1 June 2016 Accepted: 7 June 2016

Published online: 23 June 2016

\section{References}

1. Ambegaokar SS, Jackson GR. Functional genomic screen and network analysis reveal novel modifiers of tauopathy dissociated from tau phosphorylation. Hum Mol Genet. 2011;20:4947-77. doi:10.1093/hmg/ddr432.

2. Auluck PK. Chaperone suppression of alpha -synuclein toxicity in a drosophila model for Parkinson's disease. Science. 2001;295:865-8. doi:10. 1126/science.1067389.

3. Bateman RJ, Xiong C, Benzinger TLS, Fagan AM, Goate A, Fox NC, Marcus DS, Cairns NJ, Xie X, Blazey TM, Holtzman DM, Santacruz A, Buckles V, Oliver A, Moulder K, Aisen PS, Ghetti B, Klunk WE, McDade E, Martins RN, Masters CL, Mayeux R, Ringman JM, Rossor MN, Schofield PR, Sperling RA, Salloway S, Morris JC, the Dominantly Inherited Alzheimer Network. Clinical and Biomarker Changes in Dominantly Inherited Alzheimer's Disease. N Engl J Med. 2012;367:795-804. doi: 10.1056/NEJMoa1202753

4. Bendor JT, Logan TP, Edwards RH. The function of a-synuclein. Neuron. 2013;79:1044-66. doi:10.1016/j.neuron.2013.09.004.

5. Blard O, Feuillette S, Bou J, Chaumette B, Frébourg T, Campion D, Lecourtois M. Cytoskeleton proteins are modulators of mutant tau-induced neurodegeneration in Drosophila. Hum Mol Genet. 2007;16:555-66. doi:10.1093/hmg/ddm011.

6. Boassa D, Berlanga ML, Yang MA, Terada M, Hu J, Bushong EA, Hwang M, Masliah E, George JM, Ellisman MH. Mapping the subcellular distribution of asynuclein in neurons using genetically encoded probes for correlated light and electron microscopy: implications for Parkinson's disease pathogenesis. Neurosci. 2013:33:2605-15. doi:10.1523/JNEUROSCI.2898-12.2013.

7. Brand A, Perrimon N. Targeted gene expression as a means of altering cell fates and generating dominant phenotypes. Development. 1993;118:401-15.

8. Breda C, Nugent ML, Estranero JG, Kyriacou CP, Outeiro TF, Steinert JR, Giorgini F. Rab11 modulates -synuclein-mediated defects in synaptic transmission and behaviour. Hum Mol Genet. 2015;24:1077-91. doi:10.1093/hmg/ddu521.

9. Cao W, Song H, Gangi T, Kelkar A, Antani I, Garza D, Konsolaki M. Identification of novel genes that modify phenotypes induced by Alzheimer's beta-amyloid overexpression in Drosophila. Genetics. 2008;178: 1457-71. doi:10.1534/genetics.107.078394.

10. Casas-Tinto S, Zhang Y, Sanchez-Garcia J, Gomez-Velazquez M, Rincon-Limas DE, Fernandez-Funez $P$. The ER stress factor XBP1s prevents amyloid-beta neurotoxicity. Hum Mol Genet. 2011;20:2144-60. doi:10.1093/hmg/ddr100.

11. Chatterjee S, Sang T-K, Lawless GM, Jackson GR. Dissociation of tau toxicity and phosphorylation: role of GSK-3beta, MARK and Cdk5 in a Drosophila model. Hum Mol Genet. 2009:18:164-77. doi:10.1093/hmg/ddn326.

12. Chee FC, Mudher A, Cuttle MF, Newman TA, Mackay D, Lovestone S, Shepherd D. Over-expression of tau results in defective synaptic transmission in Drosophila neuromuscular junctions. Neurobiol Dis. 2005;20: 918-28. doi:10.1016/j.nbd.2005.05.029.

13. Chiang H-C, lijima K, Hakker I, Zhong Y. Distinctive roles of different betaamyloid 42 aggregates in modulation of synaptic functions. FASEB J. 2009; 23:1969-77. doi:10.1096/fj.08-121152.

14. Colodner KJ, Feany MB. Glial fibrillary tangles and JAK/STAT-mediated glial and neuronal cell death in a Drosophila model of glial tauopathy. J Neurosci. 2010;30:16102-13. doi:10.1523/JNEUROSCI.2491-10.2010.

15. Cowan CM, Quraishe S, Hands S, Sealey M, Mahajan S, Allan DW, Mudher A. Rescue from tau-induced neuronal dysfunction produces insoluble tau oligomers. Sci Rep. 2015:5:17191. doi:10.1038/srep17191.

16. Dawson TM, Ko HS, Dawson VL. Genetic animal models of Parkinson's disease. Neuron. 2010;66:646-61. doi:10.1016/j.neuron.2010.04.034.

17. Ebneth A, Godemann R, Stamer K, Illenberger S, Trinczek B, Mandelkow E. Overexpression of tau protein inhibits kinesin-dependent trafficking of vesicles, mitochondria, and endoplasmic reticulum: implications for Alzheimer's disease. J Cell Biol. 1998;143:777-94.

18. Feany MB, Bender WW. A Drosophila model of Parkinson's disease. Nature. 2000:404:394-8. doi:10.1038/35006074.

19. Fernandez-Funez $P$, de Mena L, Rincon-Limas DE. Modeling the complex pathology of Alzheimer's disease in Drosophila. Experimental Neurology. 2015;274(Pt A):58-71. doi: 10.1016/j.expneurol.2015.05.013

20. Fernandez-Funez P, Nino-Rosales M, de Gouyon B, She W, Luchak J, Martinez P, Turiegano E, Benito J, Capovilla M, Skinner P, McCall A, Canal I, Orr H, Zoghbi H, Botas J. Identification of genes that modify ataxin-1-induced neurodegeneration. Nature. 2000;408:101-6.

21. Finelli A, Kelkar A, Song $H$, Yang $H$, Konsolaki M. A model for studying Alzheimer's Abeta42-induced toxicity in Drosophila melanogaster. Mol Cell Neurosci. 2004;26:365-75. doi:10.1016/j.mcn.2004.03.001.

22. Gorsky MK, Burnouf S, Dols J, Mandelkow E, Partridge L. Acetylation mimic of lysine 280 exacerbates human Tau neurotoxicity in vivo. Sci Rep. 2016;6: 22685. doi:10.1038/srep22685.

23. Gotz J, Ittner L. Animal models of Alzheimer's disease and frontotemporal dementia. Nat Rev Neurosci. 2008;9:532-44. doi:10.1038/nrn2420.

24. Haelterman NA, Yoon WH, Sandoval H, Jaiswal M, Shulman JM, Bellen HJ (2014) A Mitocentric View of Parkinson's Disease. Annual review of neuroscience. doi: 10.1146/annurev-neuro-071013-014317

25. Hernández-Vargas R, Fonseca-Ornelas L, López-González I, Riesgo-Escovar J, Zurita M, Reynaud E. Synphilin suppresses a-synuclein neurotoxicity in a Parkinson's disease Drosophila model. Genesis. 2011;49:392-402. doi:10. 1002/dvg.20740

26. Ishihara T, Hong M, Zhang B, Nakagawa Y, Lee MK, Trojanowski JQ, Lee VM Age-dependent emergence and progression of a tauopathy in transgenic mice overexpressing the shortest human tau isoform. Neuron. 1999;24:751-62.

27. Jack CR, Knopman DS, Jagust WJ, Petersen RC, Weiner MW, Aisen PS, Shaw LM, Vemuri P, Wiste HJ, Weigand SD, Lesnick TG, Pankratz VS, Donohue MC, Trojanowski JQ. Tracking pathophysiological processes in Alzheimer's disease: an updated hypothetical model of dynamic biomarkers. Lancet Neurol. 2013:12:207-16. doi:10.1016/S1474-4422(12)70291-0.

28. Jaiswal M, Sandoval H, Zhang K, Bayat V, Bellen HJ. Probing mechanisms that underlie human neurodegenerative disease in drosophila. Annu Rev Genet. 2012;46:371-96. 
29. Kordower JH, Olanow CW, Dodiya HB, Chu Y, Beach TG, Adler CH, Halliday GM, Bartus RT. Disease duration and the integrity of the nigrostriatal system in Parkinson's disease. Brain. 2013;136:2419-31. doi:10.1093/brain/awt192.

30. Kosmidis S, Grammenoudi S, Papanikolopoulou K, Skoulakis EMC. Differential effects of Tau on the integrity and function of neurons essential for learning in Drosophila. J Neurosci. 2010;30:464-77. doi:10.1523/JNEUROSCI.1490-09.2010.

31. Landau SM, Mintun MA, Joshi AD, Koeppe RA, Petersen RC, Aisen PS, Weiner MW, Jagust WJ, Alzheimer's Disease Neuroimaging Initiative. Amyloid deposition, hypometabolism, and longitudinal cognitive decline. Ann Neurol. 2012;72:578-86. doi:10.1002/ana.23650.

32. Lees AJ, Hardy J, Revesz T. Parkinson's disease. Lancet. 2009;373:2055-66. doi:10.1016/S0140-6736(09)60492-X.

33. Lessing D, Bonini NM. Maintaining the brain: insight into human neurodegeneration from Drosophila melanogaster mutants. Nat Rev Genet. 2009;10:359-70. doi:10.1038/nrg2563.

34. Lim J, Yue Z. Neuronal aggregates: formation, clearance, and spreading. Dev Cell. 2015;32:491-501. doi:10.1016/j.devcel.2015.02.002.

35. Merlo P, Frost B, Peng S, Yang YJ, Park PJ, Feany M. p53 prevents neurodegeneration by regulating synaptic genes. Proc Natl Acad Sci USA. 2014;111:18055-60. doi:10.1073/pnas.1419083111.

36. Mershin A, Pavlopoulos E, Fitch O, Braden BC, Nanopoulos DV, Skoulakis EMC Learning and memory deficits upon TAU accumulation in Drosophila mushroom body neurons. Learn Mem. 2004;11:277-87. doi:10.1101//m.70804

37. Mollereau B, Dominguez M, Webel R, Colley NJ, Keung B, de Celis JF, Desplan C. Two-step process for photoreceptor formation in Drosophila. Nature. 2001;412:911-3. doi:10.1038/35091076.

38. Mudher A, Shepherd D, Newman T, Mildren P, Jukes J, Squire A, Mears A, Drummond J, Berg S, MacKay D, Asuni A, Bhat R, Lovestone S. GSK-3beta inhibition reverses axonal transport defects and behavioural phenotypes in Drosophila. Mol Psychiatry. 2004;9:812.

39. Nagarkar-Jaiswal S, Lee PT, Campbell ME, Chen K, Anguiano-Zarate S, Cantu Gutierrez M, Busby T, Lin W-W, He Y, Schulze KL, Booth BW, Evans-Holm M, Venken KJT, Levis RW, Spradling AC, Hoskins RA, Bellen HJ. A library of MiMIC insertion lines allows intronic EGFP tagging for reversible spatial and temporal knockdown of proteins in Drosophila. eLife. 2015;4, e05338. doi:10. 7554/eLife.05338.

40. Napoletano F, Occhi S, Calamita P, Volpi V, Blanc E, Charroux B, Royet J, Fanto M. Polyglutamine Atrophin provokes neurodegeneration in Drosophila by repressing fat. EMBO J. 2011;30:945-58. doi:10.1038/emboj. 2011.1.

41. Nemani VM, Lu W, Berge V, Nakamura K, Onoa B, Lee MK, Chaudhry FA, Nicoll RA, Edwards RH. Increased expression of alpha-synuclein reduces neurotransmitter release by inhibiting synaptic vesicle reclustering after endocytosis. Neuron. 2010;65:66-79. doi:10.1016/j.neuron.2009.12.023.

42. Nishimura I, Yang Y, Lu B. PAR-1 kinase plays an initiator role in a temporally ordered phosphorylation process that confers tau toxicity in Drosophila. Cell. 2004;116:671-82.

43. Ollmann M, Young LM, Di Como CJ, Karim F, Belvin M, Robertson S, Whittaker K, Demsky M, Fisher WW, Buchman A, Duyk G, Friedman L, Prives C, Kopczynski C. Drosophila p53 is a structural and functional homolog of the tumor suppressor p53. Cell. 2000;101:91-101. doi:10.1016/S00928674(00)80626-1.

44. Overk CR, Masliah E. Pathogenesis of synaptic degeneration in Alzheimer's disease and Lewy body disease. Biochem Pharmacol. 2014;88:508-16. doi:10. 1016/j.bcp.2014.01.015

45. Papanikolopoulou K, Skoulakis EMC. Temporally distinct phosphorylations differentiate Tau-dependent learning deficits and premature mortality in Drosophila. Hum Mol Genet. 2015;24:2065-77. doi:10.1093/hmg/ddu726.

46. Querfurth HW, LaFerla FM. Alzheimer's disease. N Engl J Med. 2010;362:329_ 44. doi:10.1056/NEJMra0909142.

47. Raghu P, Coessens E, Manifava M, Georgiev P, Pettitt T, Wood E, GarciaMurillas I, Okkenhaug H, Trivedi D, Zhang Q, Razzaq A, Zaid O, Wakelam M, O'Kane CJ, Ktistakis N. Rhabdomere biogenesis in Drosophila photoreceptors is acutely sensitive to phosphatidic acid levels. J Cell Biol. 2009;185:129-45. doi:10.1083/jcb.200807027.

48. Rister J, Razzaq A, Boodram P, Desai N, Tsanis C, Chen H, Jukam D, Desplan C. Single-base pair differences in a shared motif determine differential Rhodopsin expression. Science. 2015;350:1258-61. doi:10.1126/science.aab3417.

49. Schulz-Schaeffer WJ. The synaptic pathology of alpha-synuclein aggregation in dementia with Lewy bodies, Parkinson's disease and Parkinson's disease dementia. Acta Neuropathol. 2010;120:131-43. doi:10.1007/s00401-010-0711-0.
50. Scott DA, Tabarean I, Tang Y, Cartier A, Masliah E, Roy S. A pathologic cascade leading to synaptic dysfunction in alpha-synuclein-induced neurodegeneration. J Neurosci. 2010;30:8083-95. doi:10.1523/JNEUROSCI. 1091-10.2010

51. Sheng G, Thouvenot E, Schmucker D, Wilson DS, Desplan C. Direct regulation of rhodopsin 1 by Pax-6/eyeless in Drosophila: evidence for a conserved function in photoreceptors. Genes Dev. 1997;11:1122-31.

52. Shulman JM, Feany MB. Genetic modifiers of tauopathy in Drosophila. Genetics. 2003:165:1233-42.

53. Singleton $A B$, Farrer $M$, Johnson J, Singleton $A$, Hague $S$, Kachergus J, Hulihan M, Peuralinna T, Dutra A, Nussbaum R, Lincoln S, Crawley A, Hanson M, Maraganore D, Adler C, Cookson MR, Muenter M, Baptista M, Miller D, Blancato J, Hardy J, Gwinn-Hardy K. alpha-Synuclein locus triplication causes Parkinson's disease. Science. 2003;302:841. doi: 10.1126/science.1090278

54. Sperling RA, Jack CR, Aisen PS. Testing the right target and right drug at the right stage. Sci Transl Med. 2011;3:111 cm33. doi: 10.1126/ scitransImed.3002609

55. Sperling RA, Laviolette PS, O'Keefe K, O'Brien J, Rentz DM, Pihlajamaki M, Marshall G, Hyman BT, Selkoe DJ, Hedden T, Buckner RL, Becker JA, Johnson KA. Amyloid deposition is associated with impaired default network function in older persons without dementia. Neuron. 2009;63:178-88. doi:10. 1016/j.neuron.2009.07.003.

56. Spires-Jones TL, Hyman BT. The intersection of amyloid beta and tau at synapses in Alzheimer's disease. Neuron. 2014;82:756-71. doi:10.1016/j. neuron.2014.05.004.

57. Steinhilb ML, Dias-Santagata D, Mulkearns EE, Shulman JM, Biernat J, Mandelkow E-M, Feany MB. S/P and T/P phosphorylation is critical for tau neurotoxicity in Drosophila. J Neurosci Res. 2007;85:1271-8. doi:10.1002/jnr.21232.

58. Verstreken P, Koh T-W, Schulze KL, Zhai RG, Hiesinger PR, Zhou Y, Mehta SQ, Cao Y, Roos J, Bellen HJ. Synaptojanin is recruited by endophilin to promote synaptic vesicle uncoating. Neuron. 2003:40:733-48.

59. Walker LC, Diamond MI, Duff KE, Hyman BT. Mechanisms of protein seeding in neurodegenerative diseases. JAMA Neurol. 2013;70:304-10. doi:10.1001/ jamaneurol.2013.1453.

60. Wang T, Montell C. Phototransduction and retinal degeneration in Drosophila. Pflugers Arch. 2007;454:821-47. doi:10.1007/s00424-007-0251-1.

61. Wang X, Wang W, Li L, Perry G, Lee H-G, Zhu X. Oxidative stress and mitochondrial dysfunction in Alzheimer's disease. Biochim Biophys Acta. 2014;1842:1240-7. doi:10.1016/j.bbadis.2013.10.015.

62. Wittmann CW, Wszolek MF, Shulman JM, Salvaterra PM, Lewis J, Hutton M, Feany MB. Tauopathy in Drosophila: neurodegeneration without neurofibrillary tangles. Science. 2001;293:711-4. doi:10.1126/science.1062382

63. Xiong B, Bayat V, Jaiswal M, Zhang K, Sandoval H, Charng W-L, Li T, David G, Duraine L, Lin YQ, Neely GG, Yamamoto S, Bellen HJ. Crag Is a GEF for Rab11 Required for Rhodopsin Trafficking and Maintenance of Adult Photoreceptor Cells. PLoS Biol. 2012;10:e1001438. doi:10.1371/journal.pbio. 1001438.

64. YYamamoto S, Jaiswal M, Charng W-L, Gambin T, Karaca E, Mirzaa G, Wiszniewski W, Sandoval H, Haelterman NA, Xiong B, Zhang K, Bayat V, David G, Li T, Chen K, Gala U, Harel T, Pehlivan D, Penney S, Vissers LELM, de Ligt J, Jhangiani SN, Xie Y, Tsang SH, Parman Y, Sivaci M, Battaloglu E, Muzny D, Wan Y-W, Liu Z, Lin-Moore AT, Clark RD, Curry CJ, Link N, Schulze KL, Boerwinkle E, Dobyns WB, Allikmets R, Gibbs RA, Chen R, Lupski JR, Wangler MF, Bellen HJ. A Drosophila Genetic Resource of Mutants to Study Mechanisms Underlying Human Genetic Diseases. Cell. 2014;159:200-14. doi:10.1016/j.cell.2014.09.002.

65. Ye C, Walsh DM, Selkoe DJ, Hartley DM. Amyloid beta-protein induced electrophysiological changes are dependent on aggregation state: $\mathrm{N}$ methyl-D-aspartate (NMDA) versus non-NMDA receptor/channel activation. Neurosci Lett. 2004;366:320-5. doi:10.1016/j.neulet.2004.05.060. 